Proposal of an innovative interface for a t angi bl e communi cat $i$ on bet ween PCs and vi sual I y i mared peopl e by usi ng ai $r$ propel I ed new techni que of wi nd el ement s( wi xel)

\begin{tabular}{|c|c|}
\hline 著者 & $\begin{array}{l}\text { Rebol I edo Me?ndez Jovan Davi d, Nakayama Kenj i , } \\
\text { Mendez Mor al es Adol fo }\end{array}$ \\
\hline $\begin{array}{l}\text { jour nal or } \\
\text { publ i cat } i \text { on } \mathrm{title}\end{array}$ & $\begin{array}{l}\text { TENCON } 2008 \text { - 2008, TENCON 2008. I EEE Regi on } \\
10 \text { Conf er ence }\end{array}$ \\
\hline page $r$ ange & $1-5$ \\
\hline year & $2008-11-01$ \\
\hline URL & ht t p: //hdl . handl e. net /2297/18076 \\
\hline
\end{tabular}




\title{
Proposal of an innovative interface for a tangible communication between PCs and visually impared people by using air propelled new technique of wind elements (wixel)
}

\author{
Jovan David REBOLLEDO MÉNDEZ ${ }^{\dagger}$, Kenji NAKAYAMA ${ }^{\dagger}$, \\ Adolfo MÉNDEZ MORALES* \\ $\dagger$ †ANAZAWA UNIVERSITY \\ Graduate School of \\ Natural Science and Technology, \\ Kanazawa-shi, 920-1192 Japan \\ jovan@leo.ec.t.kanazawa-u.ac.jp http://leo.ec.t.kanazawa-u.ac.jp/ jovan/ \\ $*$ UV-ITESM \\ Graduate School in Education \\ Campus Monterrey, Sede Xalapa, Mexico
}

\begin{abstract}
Viusally impared people face many obstacles to interact properly with the current screen / visually oriented technologies, as monitors or in using Internet, being destined just for users with good visual ability. This paper refers to a specification of a proposal of an innovative way to safe that obstacle for visually impaired people and provide them a device that will make them understand the normal output from a computer. This method attemps to use the basic element: a wind element (wixel). It will be explained its technical specification, methodology, materials, usages, benefits, current developent and future work as well.
\end{abstract}

\section{Introduction}

Visual impairment, in its different degrees [1], has existed along humanity, always representing a big challenge to those affected people for not being as productive as the ones who have good visual capacity. The Braille System, devised in 1821 , is the method by which blind people rely to write and read texts, music, etc. Since the entrance of the digital computer systems, which use primarily a visual display, or monitor, as a default output to communicate to it, the gap of benefit in getting information for the visual impairment people and for the ones who are not, widen extremely.
But, thanks to other systems designed to reduce this gap, as the Braille Display (which mechanically raises dots on a board), or DAISY standard ${ }^{1}$ [4](which helps in reproducing text vocally), blind people count with some resources to get information from digital components. The most important advance for blind people has been the invention of the Braille System, which consists in 8 dots that symbolize any roman character (in English). (Fig.1-(a) shows the Braille System, and (b) gives examples of the use of this decoding system.) The representation of the dots (quantity and position) becomes a single compact unit that enables reading. A dot may be raised, or repressed, making it noticeable for the user who feels tangibly the difference between the plane and the no-plane surface. There have been different ways to provide tactual displays [7], and there have been different approaches [2], [8] and [5] that have tried to implement coding from digital information to tactile perception. In this paper the basic idea of the Braille System is amplified and taken to a different level, proposing a development of a device that provides tangible sensorial perception by small charges of air that are felt by the hand of the user, by approaching the hand to a board that has hundreds of small air projectors. Every single air projector would be small enough to simulate a single pixel (in an analogy to a visual display), but in this case being done by wind ele-

\footnotetext{
${ }^{1}$ Digital Accessible Information SYstem (DAISY) Consortium. Web page: http://www.daisy.org/
} 

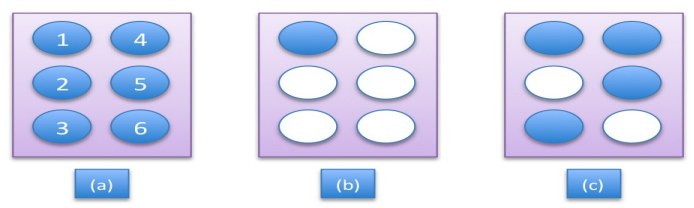

Figure 1. Braille System: (a) Braille cell, displaying the standard order of the 6 dots that represent any letter in English; (b) Braille representation: characters "A" (b), and "N" (c).

ments, or wixels. The resulting board would be executed in either a static or dynamic synchronization of the data to be displayed. In order to optimize the scarcity of definition done by not so many single wixels, the wixel displayed text would be done by Braille system, but the board would be enough to be enable to give the sensation of visual display (as in first generations of mobile phone screens). This paper describes and engages a new way to bring the output of a computer into a way of tangibly recognizing the surfaces, windows, images, and text as it were on a normal monitor by the use of small units of propelled wind (enclosed on a matrix way), which outputs small quantities of air to become a tangible understanding for a blind person. In section 2 it will be explained the proposed specification for such a device. In section 3 it will be addressed the materials and components that would be used to this approach. In section 4 it will be mentioned a proposed XML standard to visualize the ASCII character set into points, for a more focused approach to correct visualization. In section 5 it will be mentioned the current development state, and other benefits of such a device, as well as future work to be done. It will be followed by conclusions and reference materials.

\section{Wixel Technical Specification}

\subsection{Wixel Definition}

The wind element (wixel) is the basic unit of the methodology of this new communication mechanism, and intends in serving as a dynamic "visualizator" of a configurable monitor for a blind person. Its design consist of very small a pneumatic air propeller (Fig.2-(a),(b)) that can be taken as a basic unit (either in representing an image, or a braille written system's dot) on a table matrix of arranged wixels (Fig.2-(c)) that individually propels or stops the air to the outside. A physical wixel could be defined as a single air probe that has air as an input and output, but the frequency of the supplied air is controlled by a dedicated hardware that interacts between the CPU and the wixel board, synchronizing the entire board's matrix of wixels.
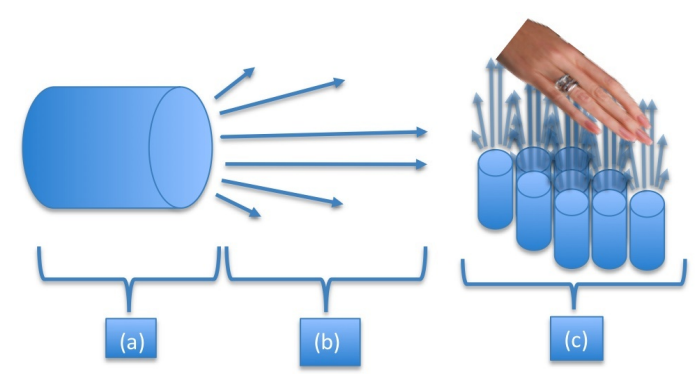

Figure 2. (a)Air probe mechanism shows air (b) propelled in a direction; (c) wixels cell arranged in a matrix made tangible by an user's hand.

\subsection{Wixel Technical Specification Proposal}

The input air is received by a switch that has multiple channels that open or close in accordance to the CPU signal from the VGA, that usually is carried to a normal monitor, to process the correspondent images. The proposed methodology could be summarized as:

1. The video card processes images information in a normal way.

2. The images are transmitted as video graphic array (VGA).

3. The VGA port connects with the switcher decoder, a Programmable Logic Controller (PLC) which reprocesses the signals into a binary (permitting or suppressing air) input for the mechanical function that provides air to the respective channel of each air probe (wixel). The mechanism of the VGA decoder for the wixel's matrix will be explained in next section. See Fig.3.

4. The wixel display resolution would be low: 40x20 wixels size, enough to permit readable text.

5. Air display unit would be composed of limited array of air probes, meaning each wixel an air probe that turns on and of, each wixel being an element of the interface (like window, frame, etc.) or a point of a letter in the braille alphabet (expanded or contracted).

6. The aspect ratio would try to be $2: 1$. A small display but enabling enough output to be read.

7. The response time would be enabled in two modes: one fixed and other variable, this sencond would let the user to change at once all the display's data that was physically read. There would be an external switch that would permit such interaction.

8. The dot pitch would not be as small as in visual displays, proposed to be around $1 \mathrm{~mm}$ (around 4 times the visual display). 


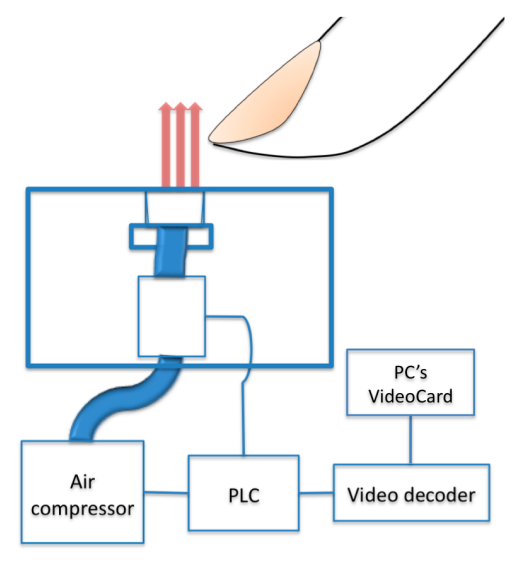

Figure 3. It can be seen the different elements of a wixel: the air exit, the air compressor, the PLC, and the Video decoder.

9. The spring force of a wixel would be in a range of 1.963.92 bars (around 20-40g).

10. The tip angle of a wixel would be between $30-90^{\circ} \mathrm{C}$.

\subsection{Wixel Board Specification Proposal}

As mentioned before, the matrix of 40 by 20 wixels with a dot pitch of $1 \mathrm{~mm}$ would consist of the next characteristics (see also Fig.3):

1. Fourty by twenty wixels, or air probes, that are aligned in a board matrix that is fixed (as shown in Fig.4).

2. Each wixel is pluged to a hose and and with connectors to the main switch-decoder, which is the decoder of the VGA, and encoder to the wixels, by selecting and letting pass or not air in accordance to the input signal to the correspondent probes.

3. A cable to be connected to a VGA in a computer.

As seen on Fig.3, the video decoder receives the output from the video card. This modules outputs to a PLC a matrix that will be represented in the wixel board. The PLC controls digitally the mechanism of the valves, which receive constant compressed air from that specific unit.

On Fig.4 shows a visualization of the way wixels would be performing in a displaying mode. The wixel board defined as a set of air probes would not have in fact any visual display, but it would permit a tangible experience that mimics the way of a visual display, but just tangible. In Fig. 4 it could be seen that the $40 \times 20$ array of wixels provide a sufficient display to read long messages. It could be adapted to read not just text but also basic images (as squares, triangles, etc.), adding the to Braille System a further experience.

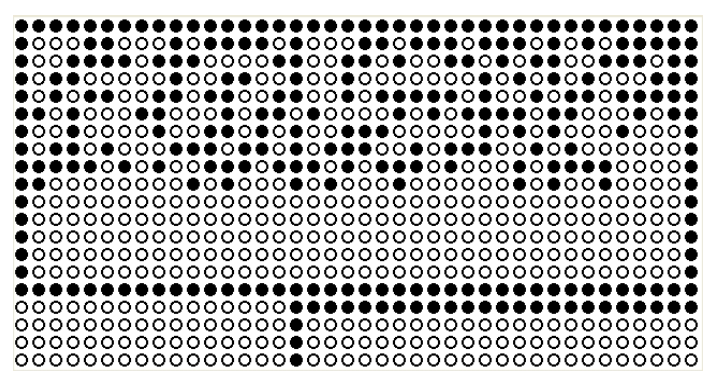

Figure 4. Simulated interface of the wixel board, consisting in a big window with the expanded Braille text of "The wixel technology is a way to understand the internet fast" inside it. It could be observed another opened "window" on the graphic as well, meaning that there would be capacity of employing more different elements like windows, or many words in braille text (expanded or contracted ), and other characteristics as interaction.

\subsection{Switcher decoder (dedicated PLC)}

The switcher decoder is being designed to be an specific Programmable Logic Control (PLC) to be able to manipulate switches that open or close the correspondent valve for letting pass or stops the current of air that flaws up to the end of the probes (wixels), previously decoded from the signal received from the VGA port. Some of the characteristics include:

1. Connection from VGA port (HD-15) by a connector (D-sub).

2. The received VGA is mapped to the correspondent switch controlled by the PLC and the one that connects to the wixel.

3. Depending of the type of sync, the device would change the configuration of those mapped switches in a different response time.

4. The device would continue sending the current coding of the received image, and switching on and off the wixels.

5. The device would control also the air compressor(s) that inject the air to the wixels, enabling more than 2 states of continuing flux of air permitting simulating the touch of a more than two-dimensional surface. 


\subsection{Interacting with the wixel board with a pointing device}

The use of a pointing device would be important to provide a feedback of the wixel display. Its use would be made in a similar way that the current mouse in a visual display when interacting with the user:

1. The presence of the mouse would be represented by four wixels syncretizing the same intermittent flow and lack of propelled air on the wixel board (i.e. with a different frequency of refreshing).

2. The clicking on would affect the elements on the same way that on a visual display.

3. The mouse also could be used to navigate into a higher dimension of display definition: the current matrix of wixel board would represent a window into the bigger set of display, so the navigation of that window could be done by making to any direction the user wants, so the current display of wixels would change as well.

\section{Materials and Components of wixel device}

The materials to be used in the design of the wixel board, and the device decoder for wixels could not represent a enormous invest in R\&D, but just using and applying current technologies as:

1. Tiny pneumatic circuits (an $\mathrm{N} \times \mathrm{M}$ air array of control and lead unit, a maintenance unit, and valves or air probes (wixels)).

2. Dedicated multi-channel air compressor.

3. D-sub connector.

4. Switchers for M x N matrix of wixels (40x20 for a simple wixel board).

5. Digital design to control the switchers. The pneumatic air probes whose size are between 1-4 mm. The switcher for controlling the $\mathrm{M} \times \mathrm{N}$ matrix of wixels is a dedicated PLC with specific functions.

\section{Proposed XML standard to direct decoding for the wixel device}

As a future effort to overcome some obstacles in the decoding of visual components into wixel displays, it is proposed in analogy to DAISY standard, which establishes a limited set of requirements for digital talking book (DTB) playback devices, whose content can range from XML text only, to text with corresponding spoken audio, to audio with little or no text. Based on SMIL 2.0 standard from WC3 [6], and taking many modules as models from it, it is the purpose to establish a XML standard for enabling the designs of any web page to be ported to an specific standard in a web page. This would permit the correct decoding of those elements of the web page into a small size wixel board specifications. The XML standard would consist (but not limited to) of:

1. Ability to structure a web page into an specific design in wixel frames of $\mathrm{X}$ by $\mathrm{Y}$ pixel-dimension.

2. Reducing excess of information that is not needed to be presented at once (for example, reducing the number of frames to a single one, and offering navigation option for both.

3. Easier representation of special characters, as well as mathematical formulas, or omitting or limiting certain design, and / or media.

4. Enable functionality and work with DAISY standards, to gain more interaction between the user and the computer.

\section{Development state, benefits and usability, and future work}

The current development state is in its second stage. This proposal is a keystone for introducing this idea and making the necessary steps into the development process that it requires. It is being detailed and analyzed the different elements and technical limitations in order to create a prototype of the product.

Once get a prototype, a battery test should be done with blind people to test its effectiveness and find challenges to overcome. The patents are being submitted. The work to be done is wide, like producing, testing, and evaluating:

1. The probes (wixels) in real world, with a functional prototype.

2. The switcher decoder (or dedicated PLC).

3. The wixel board.

4. The mouse interaction, by regulating with specific frequency the air in the wixels.

5. The XML wixel standard.

6. Production and sales for real users.

Also, future versions of the wixel board that have not just hundreds, but thousands of wixels, would permit a very close to reality experience of understanding images for visually impaired people. Also, this interaction could be used for getting data to people who are visually occupied, like pilots, giving them more information that current visual display do, or as a controller for specific video games for educational purposes. The possibilities that are open now are intended to contribute to a better world. 


\section{Conclusions}

It was described the way to create a new device capable of providing a tangible interaction with display consisting in "air dots", or wixels, that would provide a way to understand the output of the computer in a different way. This proposal gives the initial step into the development of a new way to tangibly interact between a visually impaired person and a normal output display of a computer. The wixel technology (wixel board, and switcher decoder) will provide blind people the ability to not just tangibly read the display, but also to interact with it with a mouse, with a high degree of interaction. The development of such technology would permit many people be beneficed of the knowledge that Internet can bring, and giving visual impairment people a new way to explore the world.

\section{Acknowledgements}

Part of this research was supported by the Ministry of Education, Culture, Sports, Science and Technology (MEXT), of the Government of Japan.

\section{References}

[1] AMA. "Guides To The Evaluation Of Permanent Impairment, AMA Guides - Vision Chapter”, 2001. 5th Edition.

[2] M. R. C. S. R. Bliss, J.C.; Katcher. "optical-to-tactile image conversion for the blind". IEEE Transactions on Man Machine Systems, 11(11):58-65, March 1970.

[3] P. Heckbert. "Multiresolution Surface Modeling”. SIGGRAPH 97, Los Angeles, California, August 2007.

[4] National Information Standards Organization. "Specifications for the Digital Talking Book”, April 2005.

[5] J. Pasquero and V. Hayward. "STReSS: A practical tactile display system with one millimeter spatial resolution and 700 hz refresh rate". In Proc. of Eurohaptics 2003, pages 94-110, 2003.

[6] L. Rutledge. "SMIL 2.0: XML for web multimedia". IEEE Internet Computing, 5(6):78-84, September/October 2001.

[7] H. Z. Tan and A. Pentland. "tactual displays for wearable computing". Personal and Ubiquitous Computing, 1(4):1617-4917, December 2006.

[8] V. H. Vincent Levesque, Jerome Paquero and M. Legault. "display of virtual braille dots by lateral skin deformation: Feasibility study". ACM Transactions on Applied Perception, 2(2):132-149, April 2005. 